\title{
INFESTAÇÃO EXPERIMENTAL EM GIRINOS DE RANA CATESBEIANA SHAW POR COPEPODITOS DE LERNAEA CYPRINACEA LINNAEUS (COPEPODA, LERNAEIDAE)
}

\author{
Maurício Laterça Martins ${ }^{1}$ \\ Flávio Luiz de Souza Jr. ${ }^{2}$
}

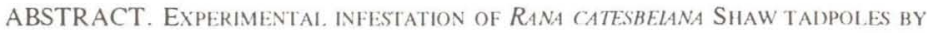
COPEPODIIS OF LERNAtA CYPRINACEA LinNaEUS (COPEPODA, LFRnAEIDAE). The Lemaea cyprinacea Linnaeus, 1758 copepodids experimental contamination on the Rana catesbeiana Shaw. 1802 tadpoles was analized. Sixty pereent (12) of infested tadpoles was found parasitized. High mortality range, lower appetence, equilibrium loss and apathy were observed. The parasites showed the preference for the mouth and cloaca: in 72 hours the egg sacs had already developed.

KEY WORDS. Copepoda, Rana catesbeiana. Lernaea cyprinacea, tadpoles, experimental infestation
\end{abstract}

O início da ranicultura intensiva no Brasil ocorreu em 1935 com a introdução de 300 casais de Rana catesbeiana Shaw, 1802 (VızotTo 1984). Daquela época até nossos dias, não houve notável preocupação quanto às conseqüências que pudessem advir em relação à disseminação de doenças em anfỉhios. Tal fato, possivelmente aliado às dificuldades no manejo, talvez seja responsável pelo relativo desenvolvimento da ranicultura no país. Deve-se associar a isto o escasso número de profissionais na matéria, número comparativamente menor em relação àqueles que se dedicam ao estudo das doenças de peixes. Por outro lado, a literatura nos tem fornecido informações que demonstram um interesse crescente quanto ao estudo de doenças de anfíbios criados em cativeiro como relatado por Glorioso et al. (1974), Araujo \& Artigas (1982), SOUZA JR. et al. (1984, 1989, 1990, 1991, 1993), SOUZA JR. (1985, 1988); BARROS et al. (1988), BAldassi et al. (1988), SOUza Jr. \& Souza (1989), Souza et al. (1990), Magalhães et al. (1991), Souza JR. \& Martins (1991, 1992), Martins \& SOUZA JR. (1995).

Algumas espécies de crustáceos ectoparasitos são importantes na piscicultura por sua notável ação patogênica em organismos aquáticos. Lernaea cyprinacea Linnaeus, 1758 é parasito próprio de peixes de água doce (PELLITERo 1988). As fêmeas, adaptadas à vida parasitária, possuem corpo alongado, cilíndrico e vermiforme. A região anterior é provida de apêndices quitinosos situados

1) Centro de Aquicultura, Universidade Estadual Paulista. Campus de Jaboticabal. Rodovia Carlos Tonanni km 05, 14870-000 Jahoticabal. São Paulo. Brasil.

2) Departamento de Parasitologia, Instituto de Ciências Bionédicas. Universidade de São Paulo. Av. Lineu Prestes 1374. 05508-000 São Paulo, São Paulo, Brasil. 
ao redor da boca e semelhantes à âncoras. Tais apêndices prendem-se fortemente aos tecidos do hospedeiro podendo em peixes pequenos, causar danos importantes caso atinjam órgãos, tais como o fígado e cérebro (PELlTtero 1988). O parasito passa por diversas fases de copepodito, semelhantes aos copépodos em geral, e após a cópula, que ocorre no sexto copepodito, o macho não é mais visto e a fêmea fertilizada continua seu desenvolvimento aumentando rapidamente de tamanho e crescendo no tegumento do órgão parasitado (POST 1987). Lernaea cyprinacea pode completar seu ciclo de vida em girinos de anfíbios (EIRAS 1994). As informações quanto ao parasitismo em girinos são escassas (CRESSEY 1983) e consideradas de pouca importância, segundo FLYNN (1973). O presente trabalho tem como objetivo o estudo da infestação experimental de girinos de rã-touro (Rana catesbeiana) com copepoditos de L. cyprinacea.

\section{MATERIAL E MÉTODOS}

Copepoditos de L. cyprinacea foram coletados nos arcos branquiais de um pacu (Piaractus mesopotanicus Holmberg, 1887) de $18 \mathrm{~cm}$ de comprimento padrão, proveniente de uma piscicultura comercial da região de Jaboticabal (São Paulo). No peixe, que apresentava hemorragias no corpo e comprometimento das funções respiratórias devido ao intenso parasitismo, foram coletados 10.333 copepoditos (Fig. 1). Os parasitos adultos (Fig. 2) mediram 10,8 $\pm 1,2 \mathrm{~mm}$. Para a identificação específica do crustáceo foram utilizados 20 espécimes do total de parasitos (61) coletados na cavidade bucal (2), opérculos (2), nadadeira dorsal (10), nadadeira adiposa (3), nadadeira caudal (20), nadadeira anal (15), nadadeiras peitorais (3) e superficie do corpo (6). O experimento desenvolveu-se no Laboratório do Centro de Aquicultura da Universidade Estadual Paulista de Jaboticabal. Para a infestação foram utilizados dois aquários com capacidade de 10 litros, com aeração e 10 girinos sadios medindo $4,8 \pm 0,47 \mathrm{~cm}$, em cada aquário. A quantidade de parasitos em cada aquário correspondeu a nove copepoditos $/ \mathrm{ml}$ de água $(90.000$ copepoditos). A temperatura da água manteve-se por volta de $22 \pm 0,3^{\circ} \mathrm{C}$. Os girinos foram coletados em 16, 24, 30, 48, 72 e 96 horas, sacrificados com éter etílico, fixados em formalina $10 \%$ tamponada por 48 horas e conservados em álcool $70 \%$. Procedeu-se a contagem de parasitos nos girinos e observação dos locais de preferência de fixação e crescimento. A coleta dos parasitos adultos nos peixes e nos girinos foi feita com o auxílio de estiletes e bisturi sob microscópio estereoscópico. Os espécimes obtidos foram fixados em álcool $70 \%$ e conservados em álcool $70 \%$ glicerinado a $10 \%$ para posterior identificação.

\section{RESULTADOS E DISCUSSÃO}

Do número total de girinos contaminados, 12 (60\%) mostraram-se infestados por L. cyprinacea (Fig. 3). Os parasitos instalaram-se principalmente na boca e cloaca (Tab. I). Nos girinos parasitados pode-se notar sinais tais como diminuição da ingestão de alimento, apatia e perda de equilibrio. Nos tecidos com instalação dos parasitos foi nítida a presença de reação inflamatória, sendo que, 


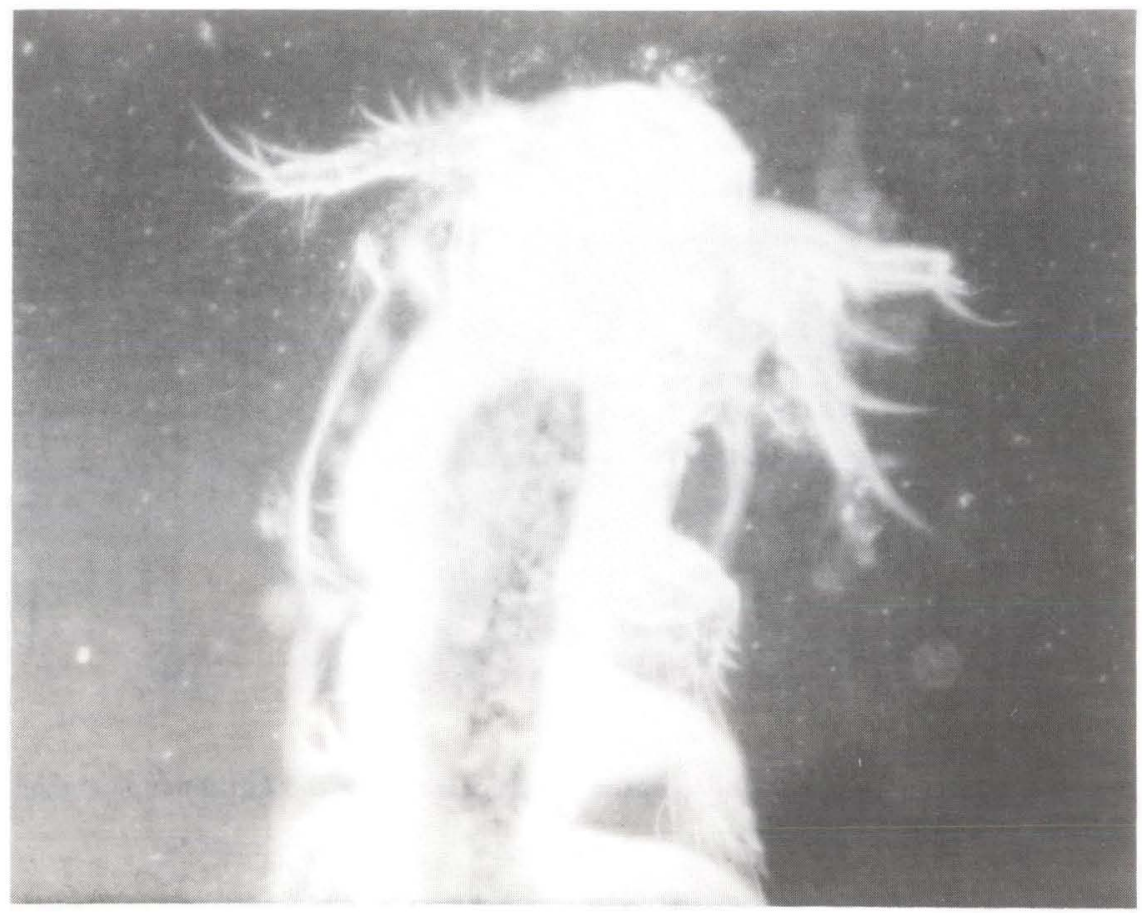

Fig. 1. Vista ventral de forma imatura (copepodito) de Lernaea cyprinacea.

Tabela I. Número, localização e tamanho de espécimes de Lernaea cyprinacea Linnaeus, 1758 coletados em girinos de Rana catesbeiana Shaw, 1802, de acordo com o tempo de infestação experimental.

\begin{tabular}{cccccc}
\hline \multirow{2}{*}{$\begin{array}{c}\text { Número total } \\
\text { de parasitos }\end{array}$} & \multicolumn{3}{c}{ Regiầ do corpo } & $\begin{array}{c}\text { Comprimento } \\
(\mathrm{mm})\end{array}$ & $\begin{array}{c}\text { Tempo de sacrificio } \\
\text { após infestação (h) }\end{array}$ \\
\cline { 2 - 4 } & Cloaca & Boca & Superficie & & \\
\hline 35 & 33 & 1 & 1 & $1,0-2,0$ & 24 \\
10 & 8 & 2 & & $1,0-2,0$ & 30 \\
30 & 18 & 10 & 2 & $2,0-3,5$ & 48 \\
38 & 29 & 9 & & $2,0-6,0$ & 72 \\
13 & 11 & 2 & & $3,0-7,0$ & 96 \\
\hline
\end{tabular}

em dois casos notou-se prolapso retal. Tais alterações já haviam sido relatadas por SHIELDS \& TIDD (1974) quando de seus estudos de L. cyprinacea em girinos de Rana pipiens e $R$. clamitans.

O crescimento dos processos cefálicos deu-se após 24 horas. Durante o período de 16 horas os copepoditos, em diferentes estadios de metamorfose, movimentavam-se ativamente por toda a superfície dos girinos. Nesta fase foi nítido o incômodo causado aos girinos pela grande quantidade de parasitos, o que resultou em excessiva produção de muco na superfície externa dos espécimes parasitados. O aparecimento de sacos ovígeros iniciou-se após 72 horas. Após este período de tempo não foi possível acompanhar o desenvolvimento dos copépodos 


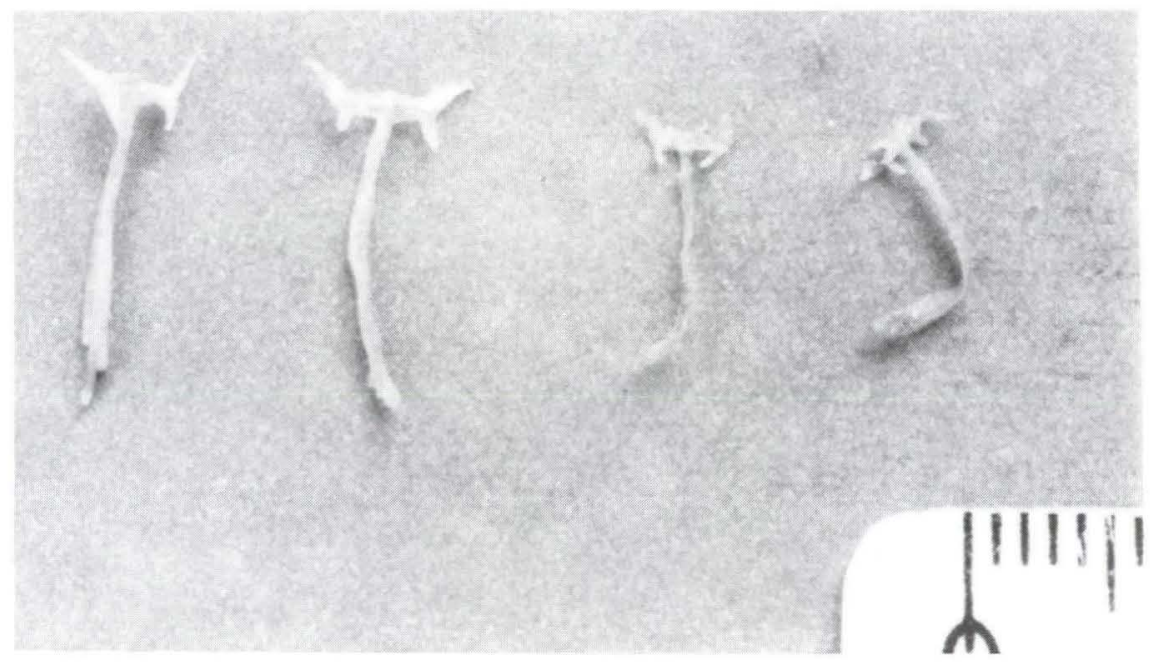

Fig. 2. Espécimes adultos do ectoparasito Lernaea cyprinacea $(10,8 \pm 1,2 \mathrm{~mm}$ de comprimento). Escala em milímetros.

em vista da morte de todos os girinos parasitados entre 72 e 96 horas; a alta taxa de mortalidade talvez esteja associada à grande perda de sangue decorrente da hiperinfestação dos girinos. De acordo com BALDAUF (1961) após coleta de girinos no Texas, para observação em aquários, $o$ autor notou o crescimento de estruturas "filamentosas" na boca e que foram identificadas como sendo copépodos parasitos denominados L. cyprinacea. Semelhante relato já havia sido mencionado por TIDD (1962) e TIDD \& SHIELDS (1963). Ainda segundo os mesmos autores, o grau de injúria depende do local parasitado no qual crescem os processos cefálicos, pois os parasitos podem alcançar os pulmões, fígado, coluna vertebral e principalmente os rins, onde atuam mecanicamente comprimindo os tecidos. SHIELDS \& TIDD (1963) observaram em girinos de $R$. catesbeiana que após 10 dias os parasitos situavam-se no espiráculo, boca ou câmara branquial. SHIELDS \& TIDD (1974), em suas observações sobre infestação de $R$. pipiens e $R$. clamitans por $L$. cyprinacea, mostraram o desenvolvimento de processo inflamatório bucal e de câmara branquial decorrente da invasão progressiva dos tecidos parasitados. Nossas observações foram compatíveis com aquelas relatadas pelos autores citados. Nos girinos, com taxas mais elevadas de infestação (Tab. I) foram nítidas as alterações teciduais cloacais, resultantes da instalação dos copépodos. Observou-se obstrução mecânica, prolapso retal acompanhado de má-formação dos membros posteriores que mostraram-se atrofiados. Alterações teciduais como inchaço da região ventral da boca não foram tão evidenciados como as lesões cloacais. O baixo índice de fixação dos copépodos nas demais superfícies dos girinos talvez possa ser explicado pela ausência de estruturas que possam atuar como suporte físico para os parasitos. Após 96 horas, o número de parasitos 


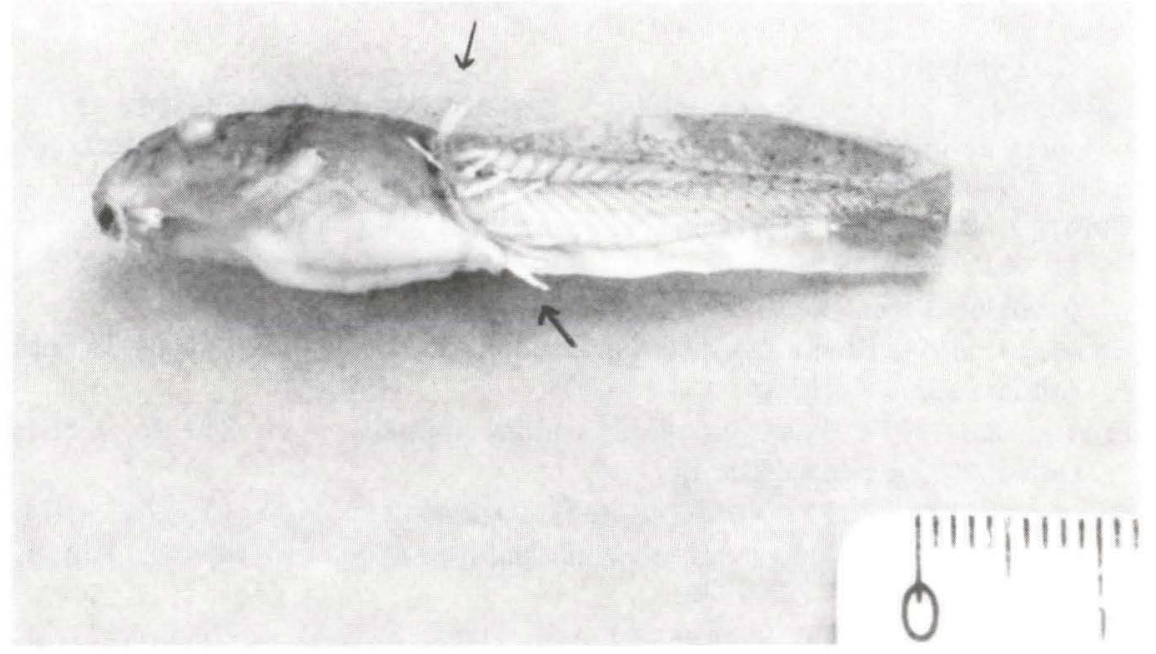

Fig. 3. Vista lateral de girino de Rana catesbeiana infestado por Lernaea cyprinacea (setas) Escala em milímetros.

encontrados nos girinos passou a diminuir devido ao número menor de hospedeiros disponíveis no aquário, tal fato ocorre devido a estes copépodos serem parasitos obrigatórios e decorrido algum tempo, sem encontrar hospedeiro vêm a morrer.

Lernaea cyprinacea é um ectoparasito próprio de peixes de água doce. Sua adaptabilidade parasitária em relação à girinos de anfíbios vem mostrar a importância que tais invertebrados exercem principalmente em relação à criação de rãs em confinamento. Instalações apropriadas e manejo adequado (MARTINS \& SOUZA JR. 1995) são as principais medidas profiláticas que previnem o contato por via hídrica dos girinos criados em ranários com animais silvestres, sejam peixes ou anfíbios.

AGRADECIMENTO. Somos gratos à Profa Marta Verardino de Stefani do Departamento de Zootecnia de não Ruminantes, Universidade Estadual Paulista (Jaboticabal, São Paulo) pelo fornecimento de girinos sadios.

\section{REFERÊNCIAS BIBLIOGRÁFICAS}

Araujo, P. \& P.T. Artigas. 1982. Gyrinicola chabaudi n.sp. (Nematoda: Pharyngodonidae) oxiurídeo encontrado em girinos. Mem. Inst. Butantan 44/45: 383-390.

Baldassi, L.; M. Hipolito; F.L. SouZa JR. \& C.W.O. SouZA. 1988. Presença de Clostridia em lesões de miŕase bucal em rã-touro gigante (Rana catesbeiana). Arq. Inst. Biol. 55: 21.

Baldauf, R.J. 1961. Another case of parasitic copepods on anphibians. J. 
Parasitol. 47: 195.

Barros, G.C.; C.H. Langenegger; J. Langenegger \& P.V. Peixoto. 1988. Surto de micobacteriose em criação de rãs (Rana catesbeiana) causado por Mycobacterium marinum. Pesq. Vet. Bras. 8 (3/4): 75-80.

Cressey, R.F. 1983. Crustaceans as parasites of other organisms, p. 251-271. In: A.J. Provenzano JR. (ed.). The biology of crustacea. New York, Academic Press, vol.6, 290p.

EIRAS, J.C. 1994. Elementos de Ictioparasitologia. Portugal, Ed. Fundação Eng. António Almeida, 339p.

FLynn, R.J. 1973. Parasites of laboratory animals. Iowa, The Iowa State University Press/Ames, 884p.

Glorioso, J.C.; R.L. Amborski; G.F. Amborski \& D.D. Culley. 1974. Microbiological studies on septicemic bullfrogs (Rana catesbeiana). Am. J. Vet. Res. 35 (9): 1241-1245.

Magalhāes, H.; G.M. Carneiro; A.R. Pires; M.E.M. Medeiros; W.M. Gonçalves; M.C. Silva; L.S. Fonseca; S.S.F. Santiago \& F.S.C.R.P. MELlo. 1991. Micobacteriose em rãs-touro (Rana catesbeiana Shaw, 1802).

Rev. Brasil. Microbiol. 22 (3): 204.

Martins, M.L. \& F.L. Souza JR. 1995. Controle de crustáceos parasitos de girinos de Rana catesbeiana Shaw, 1802. Vet. Zoot. 95.

Pellitero, P.A. 1988. Enfermedades producidas por parasitos en peces, p.215326. In: J.E. DE los Monteros \& U. Labarta (ed.). Caicyt-Plan de Formacion de Tecnicos Superiores en Acuicultura, Madrid, Ed. MundiPrensa, 550p.

Post, G. 1987. Textbook of Fish Health. New Jersey, TFH Publ., 288p.

SHIELDS, R.J. \& W.M. TIDD. 1963. The tadpole as a host for larval L. cyprinacea. J. Parasitol. 49: 1-43.

1974. Site selection on hosts by copepodids of Lernaea cyprinacea L. (Copepoda). Crustaceana 27 (3): 225-230.

SouzA, C.W.O.; R. Macruz; M.L. Martins \& F.L. Souza JR. 1990. Ocorrência de lesões cutâneas na região do crânio de imagos de rã-touro (Rana catesbeiana Shaw, 1802). Histopatologia. Resumos do I Encontro Brasileiro de Patologia de Organismos Aquáticos, São Paulo, p. 31.

Souza JR., F.L. 1985. Morfologia e ciclo vital de Giardia agilis Hunstler, 1882, em exemplares de Rana catesbeiana Shaw, 1802, de ranário comercial do Estado de São Paulo, Brasil. Tese de Doutorado, não publicada, Instituto de Ciências Biomédicas, Universidade de São Paulo, São Paulo, 82p.

1988. Doenças Parasitárias de Anfíbios. Anais do VI Encontro Nacional de Ranicultura, p.334-340.

Souza JR., F.L. \& C.W.O. Souza. 1989. Enfermidades de Rãs, p.133-143. In: S.L. Lima \& C.A. Agostinho (ed.). A criação de Rãs. Rio de Janeiro, Globo, $2^{\text {a }}$ ed, 187p.

Souza JR., F.L.; C.W.O. Souza; M. Hipolito; L. Baldassi \& M.L. Martins. 1989. Cases of buccal myiasis in the bullfrog (Rana catesbeiuna Shaw, 1802), 
with larvae of Notochaeta sp. Aldrich, 1916 (Diptera: Sareophagidae) in São Paulo, Brazil. Mem. Inst. Oswaldo Cruz 84: 517-518.

SoUza JR., F.L.; C.W.O. SouZA \& M.L. MARTINS. 1990. Gyrinicola chabaudi Araujo \& Artigas, 1982 (Nematoda: Pharyngodonidae). Description of male specimens collected from tadpoles. Rev. Brasil. Biol. 51 (3): 585-588.

Souza JR., F.L.; S. Hyakutake \& A.A. ReIS. 1984. Giardíase: interessante protozoose intestinal em girinos de rãs que afeta exemplares de Rana catesbeiana Shaw, 1802, de ranário do Estado de São Paulo, Brasil. Nota prévia. Anais do IV Encontro Nacional de Ranicultura, Goiania, p. 193-195.

Souza JR., F.L. \& M.L. Martins. 1991. Gyrinicola chabaudi Araujo \& Artigas, 1982 (Nematoda: Pharyngodonidae). Estudo do desenvolvimento pós-embrionário de exemplares coletados em formas imaturas de anfíbios. Rev. Inst. Med. Trop., São Paulo, 33 (res.): 61. 1992. Parasitismo de leptodactilídeos silvestres por vermes cosmocercídeos, capturados em ranário comercial do Estado de São Paulo. Anais do VII Encontro Nacional de Ranicultura, Rio de Janeiro, p.279.

Souza JR., F.L.; M.L. Martins \& C.W.O. SouzA. 1991. Gyrinicola chabaudi Araujo \& artigas, 1982 (Nematoda: Pharyngodonidae). Aspectos morfobiométricos de exemplares adultos coletados em formas imaturas de anfíbios. Rev. Inst. Med. Trop., São Paulo, 33 (res.): 1-61.

Souza JR., F.L.; P. Artigas \& M.L. Martins. 1993. Longibucca catesheianae n.sp. (Nematoda: Cylindrocorporidae), gastrintestinal parasite of the bullfrog (Rana catesheiana Shaw, 1802) in Brazil. Res. Rev. Parasitol. 53 (3/4): 92-102.

TIDD, W.M. 1962. Experimental infestations of frog tadpoles by Lernaea cyprinacea. J. Parasitol. 48 (6): 870.

TIDD, W.M. \& R.J. ShIELDS. 1963. Tissue damage inflicted by Lernaea cyprinacea Linnaeus, a copepod parasitic on tadpoles. J. Parasitol. 49: 693-696.

VizotTo, L.D. 1984. Ranicultura. Ciência \& Cultura 36 (1): 42-45.

Recebido em 27.II.1995; aceito em 06.X.1995. 